\title{
Can Electric Field Induced Energy Gaps In Metallic Carbon Nanotubes?
}

\author{
Xin Zhou ${ }^{1}, \mathrm{Hu} \mathrm{Chen}^{2}$ and Ou-Yang Zhong-can ${ }^{1,2}$ \\ ${ }^{1}$ Institute of Theoretical Physics, The Chinese Academy of Sciences, P. O. Box 2735, Beijing 100080, China \\ ${ }^{2}$ Center for Advanced Study, Tsinghua University, Beijing 100084, China
}

(July 15, 2021)

The low-energy electronic structure of metallic singlewalled carbon nanotube (SWNT) in an external electric field perpendicular to the tube axis is investigated. Based on tightbinding approximation, a field-induced energy gap is found in all $(n, n)$ SWNTs, and the gap shows strong dependence on the electric field and the size of the tubes. We numerically find a universal scaling that the gap is a function of the electric field and the radius of SWNTs, and the results are testified by the second-order perturbation theory in weak field limit. Our calculation shows the field required to induce a $0.1 \mathrm{eV}$ gap in metallic SWNTs can be easily reached under the current experimental conditions. It indicates a kind of possibility to apply nanotubes to electric signal-controlled nanoscale switching devices.

PACS numbers: 71.20.Tx

The prospect of nanoscale electronic devices has engaged great interest, because it could lead to conceptually new miniaturization strategies in the electronics and computer industry. Single-walled carbpn nanotubes (SWNTs) can be used as nanoscale devicest due to their extraordinarily small diameter and versatile electronic propertiest. It is suggested that individual SWNT mat act as devices such as field-effect transistors (EET) single-electpon-tunneling transistors 4 , rectifierd 6 , or p$\mathrm{n}$ junctions $\mathrm{8}$. The most exciting expectancy lies in the devices fabricated on a single tube 9 .

In recent years, the interplay between mechanical deformation and electrical properties of SWNTs have been extensively studiede 2 . Among them, some structural deformations such as twisting, bending, stretching, and topological defects are not compatible with desizable stable contacts for reversiblycontrollable devices $\mathbf{1 3}$. Very recently, Tombler et al 14,15 used an atomic force microscope tip to manipulate a metallic SWNT, leading to a reversible two-order magnitude change of conductance, and Lammert et al 13 applied a uniaxial stress to squash SWNTs and detect a similar reversible metal-insulator (M-I) transition. Since the tube ends do not need to move and they are easily controlled, the studies pointed to the possibility that a metallic SWNT may be used as an ultrasmall electromechanical switch.

It is also well known that a magnetif field can change the conductance of carbon nanotubes 16 18. The magnetic field either parallel or perpendicular to the axis can change the low-energy electronic properties of the tubes. However, a possible electric field-controlled M-I transition are more exciting because of its easy implementation in the actual applications. Now, a question remains: Can electric field change the electronic properties - f a tube?

In previous studies on electronic transports 19 , since the bias voltage is a slow-change variable in the range of the primitive unit cell, the possible change of the energyband structure induced by the bias voltage is neglected. The controlled potential, such as the gate voltage in the case of FETB, does not induce a voltage drop in the direction perpendicular to the tube axis, and it only shifts Fermi level or changes the carrier concentration. In the literature, according to our knowledge, it has not been studied on that the properties of the longitudinal electronic transports are changed by a transverse electric field. In fact, because of the small diameter of SWNTs ( about $1 \mathrm{~nm}$ ), it is easy to exert a strong electric field $\left(|\vec{E}| \sim \mathrm{V} / \mathrm{nm} \sim 10^{8-9} \mathrm{~V} / \mathrm{m}\right)$ perpendicular to the axis. In $(n, n)$ metallic SWNT, the electrons near Fermi energy are nonlocal in the circumference of the tube since their circumference Fermi wavevector is zerda, the classic wave-package approximation in slow-change voltage may be not suitable in the presence of the strong transverse electric field. The $\mathrm{V} / \mathrm{nm}$ order electric field is enough to obviously break the rotational symmetry about the tube axis, and create new interband and inter-wavevector coupling, which may change the low energy electronic properties of SWNTs, and hence affect the electronic transport. In the other hand, the field is still $2 \sim 3$ orders less than the atomic interior electric field, can be treated as perturbation.

In this Letter, based on a tight-binding (TB) model, we calculate the low-energy electronic structure of SWNTs in an external electric field perpendicular to the tube axis (see Fig.1). The result shows obviously valuable effects: (1) The electric field can always induce an energy gap in $(n, n)$ metallic SWNTs; (2) There is a maximal gap strongly dependent on the radius of the tubes; (3) A universal scaling is found for the gap as a function of the field and the size of the tubes; (4) Using the dielectric function of tubes obtained by Benedict et al.20, we find the magnitude of the required electric field falls into the range of current experimental conditions, therefore the possibility of applying SWNTs to the electric-field-controlled nanoscale switching devices.

The nearest-neighbor TB approximation has been used successfully for calculating the electronic structure of graphite sheet and nanotubes 2 , and the polarization of SWNT22 . For the low-energy electronic properties of tubes in the presence of electric field, we willgnly use the $\pi$-electron single-orbital TB approximation 21 . 
In the presence of a transverse electric field $\mathbf{E}$, there is an additional coupling between the nearest-neighbor atoms, reflected in the TB Hamiltonian

$$
\mathcal{H}=\mathcal{H}_{0}+\mathcal{H}_{1}
$$

where $\mathcal{H}_{0}$ is the unperturbed Hamiltonian, and $\mathcal{H}_{1}=$ $e V(\mathbf{r})$. Here $e$ is the charge of an electron, and $V(\mathbf{r})$ is the electrostatic potential of the total electric field which equals to the sum of an external field $\mathbf{E}_{0}$ and the polarized field induced by $\mathbf{E}_{0}$. For a uniform fielde22, the potential in the cylindrical surface of SWNT is

$$
V(\mathbf{r})=-V_{0} \cos \phi
$$

where $V_{0}=R E$ is the transverse voltage drop of the tube, and $\phi$ is the azimuth of the cylinder. Here $R$ is the radius of SWNT, and $E$ is the total electric field strength.

The main role of the electrostatic potential is to change the electronic energy of the $i$ th carbon atom by $\mathrm{eV}\left(\mathbf{R}_{i}\right)$, where $\mathbf{R}_{i}$ is the position vector of the $i$ th atom. The hopping correction due to the electric field between site $i$ and site $j$ is very small, about the order of $s$. Here $s$ is the overlap integral of two nearest peighbor atom $i$ and atom $j, s=\int \phi_{i}^{*}(\mathbf{r}) \phi_{j}(\mathbf{r}) d^{3} \mathbf{r} \approx 0.12923$, where $\phi_{i}(\mathbf{r})$ is the $\pi$-electron wavefunction of the $i$ th atom. The perturbed Hamiltonian matrix elements are:

$$
\begin{aligned}
& <\phi_{i}\left|\mathcal{H}_{1}\right| \phi_{i}>=e V\left(\mathbf{R}_{i}\right), \\
& <\phi_{i}\left|\mathcal{H}_{1}\right| \phi_{j}>\approx \operatorname{esV}\left(\mathbf{R}_{i j}^{c}\right)
\end{aligned}
$$

where $\mathbf{R}_{i j}^{c}=\left(\mathbf{R}_{i}+\mathbf{R}_{j}\right) / 2$ is the position of the mass center of the two nearest neighbor $i$ atom and $j$ atom. Eq.(3) is precise because of the symmetry of wavefunction, but in Eq.(4), we have neglected the electrostatic potential change in the overlapping range of electronic clouds. After gaining these Hamiltonian matrix elements, we can easily calculate the electronic structure of nanotube in the presence of electric field.

Fig. 2 shows our result on the electronic energy bands of $(10,10)$ tube in the presence of electric fields with different magnitudes. When $V_{0}=0.5 \mathrm{~V}$, a gap of $E_{g} \sim$ $0.3 \mathrm{eV}$ is found at $K_{0}$, the Fermi wavevector in zero field. As $V_{0}$ increases, the gap increases, and when $V_{0}=1.5 \mathrm{~V}$, we find that the bands structure is obviously deformed. It is surprising to find that the gap decreases as $V_{0}$ increases further. When $V_{0}=3.0 \mathrm{~V}$, the zero gap is found, but the Fermi point dramatically moves to a different position from $K_{0}$. The results reveal that a controlled electric field of $1 \mathrm{~V} / \mathrm{nm}$ order can obviously affect the transport properties of $(10,10)$ tube.

To probe the above effect in general, we performed the computation for a series of $(n, n)$ tubes. Fig. 3 shows the gap as a function of the electric field in $(n, n)$ tubes, where $n$ is from 5 to 15 . From the figure, we find the determined effect: The electric field can always induce a gap, i.e. a metal-insulator transition, in $(n, n)$ tubes, and the size of the gap strongly depends on the magnitude of the transverse field and the tube parameter $n$. For any $(n, n)$ tubes, the gap first increases with increasing field, and reaches a maximal value $E_{g m}$ at the $V_{0}=V_{0 m}$, then drops again. Both the maximal gap $E_{g m}$ and the corresponding $V_{0 m}$ are approximately proportional to $1 / n$, and hence inverse proportional to the radius of tubes, i.e.,

$$
\begin{aligned}
E_{g m} & \approx 6.89 \mathrm{eV} / n, \\
V_{0 m} & \approx 12.09 \mathrm{~V} / n .
\end{aligned}
$$

The finding, which is shown in (5) and (6) that the maximal gap $E_{g m}$ fastly decrease as the size of conductor increases, indicates the electronic structure change is very small in the large materials even though at very strong electric field. It might be the reason why people have not yet recognized the effect in previous studies on large conductors. In the strong field range, Fig. 3 shows a more complicated field dependence of the gap which can even be negative. The field dependence of the gap is quite similar for tubes with various radii, which invokes us to scale both $E_{g}$ and $V_{0}$ up $n$ times their original values. The obtained results are shown in Fig.4. From it we do find the scaled gap to be a universal function of the scaled electric field for all $(n, n)$ tubes. In the weak field range, for all calculated eleven $(n, n)$ tubes there exists a simple relation: $n E_{g}=\lambda\left(n e V_{0}\right)^{2}$, where $\lambda$ is a constant, about $0.07(\mathrm{eV})^{-1}$. In the middle field range, except for a few small-radius tubes such as $(5,5)$ and $(6,6)$ tubes, the universal scaling law still holds.

To understand the above scaling relation, we use perturbation theory to calculate the field-induced gap in weak field limit. The first-order perturbation approximation only causes shift in the Fermi level, showing no contribution to the gap. Calculating up to the secondorder perturbation at $K_{0}$ point, we obtained the following analytic result

$$
E_{g} \approx \frac{\sqrt{3}}{2 \pi \gamma} n e^{2} V_{0}^{2},
$$

where $\gamma(=3.033 \mathrm{eV})$ is the hppping parameter in the absence of the electric field2324. The contribution of the overlap integral $s$, which is very small, is neglected. Obviously, the second-order perturbation calculation gives almost the same scaling relation as the numerical results in the weak field, though the obtained $\lambda \approx 0.09(\mathrm{eV})^{-1}$ is slightly larger than the numerical result $0.07(\mathrm{eV})^{-1}$. In the strong field range, since the Fermi wavevector is away from $K_{0}$, the perturbation theory becomes not suitable. In fact, the weak field range may be more compatible with the practical application. In order to open a $0.1 \mathrm{eV}$ gap in the energy bands of $(n, n)$ tubes, $n$ must be smaller than 68 according to eq.(5), and the required electric field is,

$$
E=\frac{2 \pi}{3 r_{0} e} \sqrt{\frac{E_{g}}{\lambda}} n^{-\frac{3}{2}},
$$

where $r_{0}(=1.42 \AA)$ is the bond length of carbon atoms in SWNT. Therefore, for example for a $(10,10)$ tube, the 
required field is about $5 \times 10^{8} \mathrm{~V} / \mathrm{m}$, and for a $(60,60)$ tube, it is about $3 \times 10^{7} \mathrm{~V} / \mathrm{m}$. Since the total field $\mathbf{E}$ equals to the sum of the external field $\mathbf{E}_{0}$ and the of the polarized field, we have $\mathbf{E}=\mathbf{E}_{0} / \epsilon$, where $\epsilon$ is the dielectric function. Under the homogeneous polarization approximation, Benedict et al.20 calculated the $\epsilon$ of some tubes within random phase approximation. Based on their results, we have25,

$$
\epsilon=1+5.2 \frac{R^{2}}{(R+\delta R)^{2}},
$$

where $R$ is the radius of tubes. Benedict gave $\delta R$ about $1.2 \AA$. So the required external field is about 6 times of the calculated total field $\mathbf{E}$. Since such a magnitude of the electric field can be easily reached by the current experimental conditions, we wish the above prediction can be checked in near future.

In summary, we have proposed an electric-field-induced M-I transition in $(n, n)$ SWNTs for the first time. The universal relationship between the gap and the electric field has been obtained in SWNTs by using the TB model. The results support the argument that SWNTs can be applied as nanoscale electric signal-controlled switching devices.

The authors would like to thank Prof. H.-W. Peng, Prof. Z.-B. Su and Dr. H.-J. Zhou for many discussions on the results. The numerical calculations were performed partly at ITP-Net and partly at the State Key Lab. of Scientific and Engineering Computing.

${ }^{1}$ C. Dekker, Phys. Today 52 (5), 22 (1999).

N. Hamada, S. Sawada, and A. Oshiyama, Phys. Rev. Lett.
68, 1579 (1992).

${ }^{3}$ S. J. Tans, A. R. M. Verschueren, and C. Dekker, Nature (London) 393, 49 (1998).

${ }^{4}$ S. J. Tans et al., Nature (London) 386, 474 (1997).

${ }^{5}$ M. Bockrath et al., Science 275, 1922 (1997).

${ }^{6}$ Z. Yao, H. W. C. Postma, L. Balents, and C. Dekker, Nature (London) 402, 273 (1999).

${ }^{7}$ M. S. Fuhrer et al., Science 288, 494 (2000).

${ }^{8}$ F. Léonard, and J. Tersoff, Phys. Rev. Lett. 83, 5174 (1999).

${ }^{9}$ L. Chico et al., Phys. Rev. Lett. 76, 971 (1996).

${ }^{10}$ A. Bezryadin, A. R. M. Verschueren, S. J. Tans, and C. Dekker, Phys. Rev. Lett. 80, 4036 (1998).

${ }^{11}$ V. Crespi et al., Phys. Rev. Lett. 79, 2093 (1997).

12 C. L. Kane and E. J. Mele, Phys. Rev. Lett. 78, 1932 (1997).

${ }^{13}$ P. E. Lammert, P. Zhang, and V. H. Crespi, Phys. Rev. Lett. 84, 2453 (2000).

${ }^{14}$ T. W. Tombler et al., Nature (London) 405, 769 (2000).

${ }^{15}$ L. Liu et al., Phys. Rev. Lett. 84, 4950 (2000).

${ }^{16}$ H. Ajiki and T. Ando, J. Phys. Soc. Jap. 62, 1255 (1993). the axis of SWNT.
17 J. P. Lu, Phys. Rev. Lett. 74, 1123 (1995).

${ }^{18}$ S. Roche and R. Saito, Phys. Rev. B 59, 5242 (1999).

${ }^{19}$ S. Datta, Electronic Transport In Mesoscopic Systems, Combridge Univ. Press, Combridge, 1995.

${ }^{20}$ L. X. Benedict, S. G. Louie, and M. L. Cohen, Phys. Rev. B 52, 8541 (1995).

${ }^{21}$ The details of the TB approximation in the precense of electric field can be found in Refs.20, the coupling between $\sigma$ - and $\pi$ - electrons induced by the curvature effects of tubes is very small.

${ }^{22}$ We suposed the polarization of tubes is uniform 20 , the a uniform external field induce a uniform polarized field.

${ }^{23}$ X. Zhou, J.-J. Zhou, and Z.-C. Ou-Yang, Phys. Rev. B 62, 13692 (2000).

${ }^{24}$ C. T. White, D. H. Robertson, and J. W. Mintmire, Phys. Rev. B 47, 5485 (1993); H. Yorikawa and S. Muramatsu, Phys. Rev. B 52, 2723 (1995).

${ }^{25} \epsilon=\alpha_{0} / \alpha=1+\frac{2 \alpha_{0}}{R_{e f f}^{2}}$, where $\alpha_{0}$ and $\alpha$ are the unscreened and screened polarizability of tubes, respectively. $R_{\text {eff }}=$ $R+\delta R$ is the effective radius of tubes due to the screened charge is out of the wall of tubes. Benedict $t$ al. showed $\alpha_{0} \approx 2.6 R^{2}$ for all tubes ( See Fig.2 of Refs 20 ).

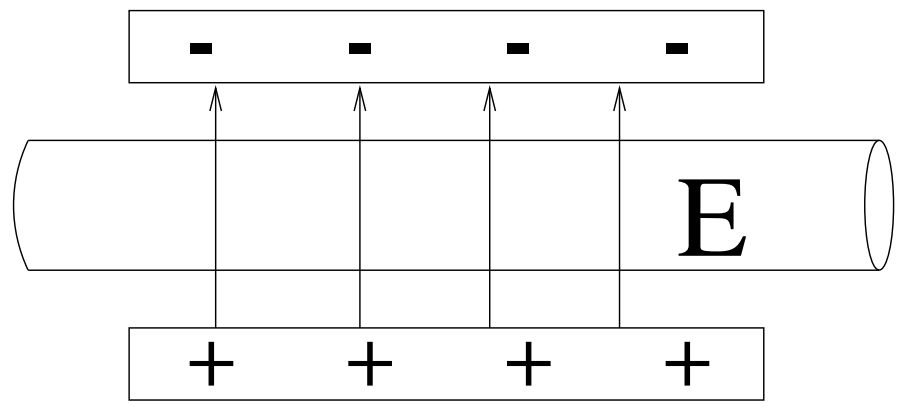

FIG. 1. A uniform external electric field perpendicular to 


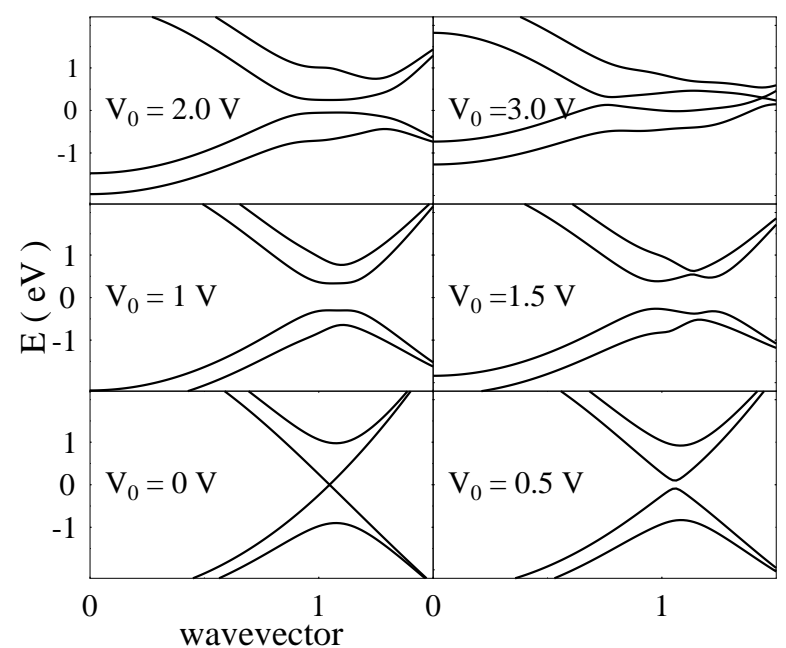

Fig.2 X. Zhou et al

FIG. 2. The energy bands of $(10,10)$ SWNT in the vicinity of Fermi level under the application of a transverse electric field $V_{0} .(10,10)$ tube is metal at $V_{0}=0 \mathrm{~V}$, and it is a semiconductor in the weak field range.

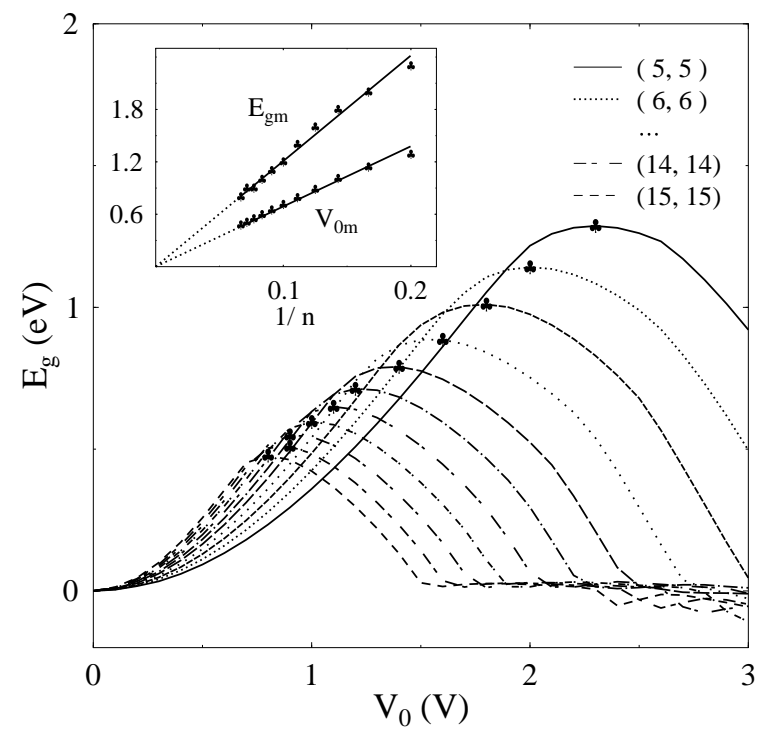

\section{Fig.3 X. Zhou et al}

FIG. 3. Field-induced gap of $(n, n)$ tubes versus the field. From the top to the bottom, the tube parameter $n$ increases from 5 to 15 . The clubs denote the position of the maximum gap point. In the inset, both the maximum gap $E_{g m}$ and $V_{0 m}$ ( see Text ) are found to be proportional to $1 / n$ The lines are fitting results.

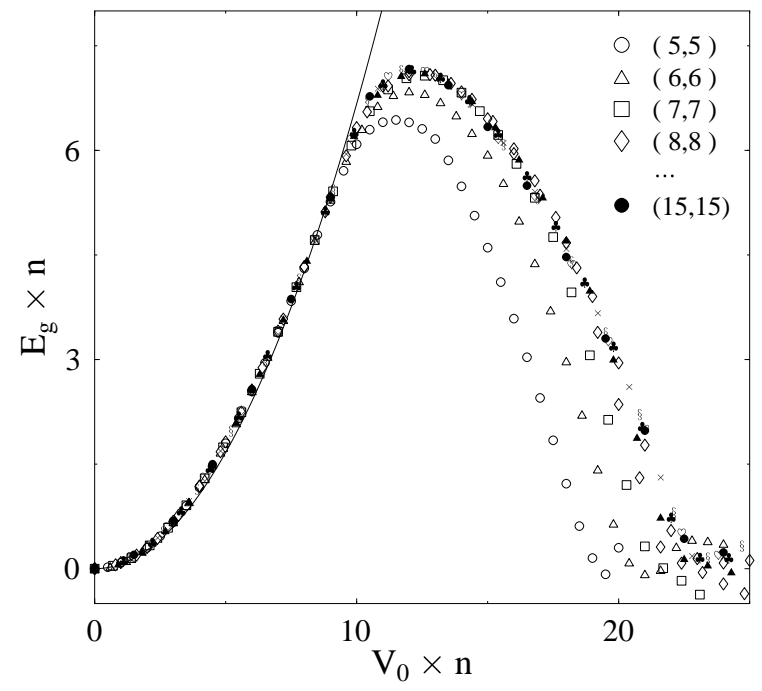

Fig.4 X. Zhou et al

FIG. 4. Universal scaling are found for the gap as a function of $V_{0}$ in different $(n, n)$ tubes. At weak field, the data of all tubes are very much consistent with the scaling relation $n E_{g}=\lambda\left(n e V_{0}\right)^{2}$, as expected by the second-order perturbation theory. The line is the fitting result. Except for $(5,5)$ and $(6,6)$ tubes, the universal scaling is satisfied well up to strong field region. 